\title{
LA COMPETENCIA DE LOS TRIBUNALES ESPAÑOLES EN LOS CONTRATOS INTERNACIONALES DE DISTRIBUCIÓN: EL AUTO DE LA AUDIENCIA PROVINCIAL DE OVIEDO DE 25 DE MAYO DE 2020
}

\author{
SPANISH COURTS JURISDICTION IN INTERNATIONAL \\ DISTRIBUTION CONTRACTUAL CLAIMS: THE AUTO COURT \\ OF APPEAL OF ASTURIAS OF 25TH MAY 2020
}

\author{
NATIVIDAD GoÑI URRIZA \\ Profesora Titular de Derecho Internacional Privado \\ Universidad Pública de Navarra \\ ORCID ID: 0000-0003-0119-3249
}

Recibido: 18.11.2020 / Aceptado: 04.12.2020

DOI: https://doi.org/10.20318/cdt.2021.6000

\begin{abstract}
Resumen: La Audiencia Provincial de Oviedo decide sobre la competencia judicial internacional y la competencia territorial en un litigio relativo a un contrato de distribución que debía ejecutarse en España. El juzgado de primera instancia se había declarado incompetente al haber entendido aplicable el art. 51 LEC por remisión del Reglamento Roma I. La Audiencia Provincial identifica la cuestión de Derecho Internacional Privado en la competencia judicial internacional y para resolver si el juzgado de Castropol es competente se plantea, en primer lugar, el ámbito de aplicación material del Reglamento Bruselas I bis. En segundo lugar, se califican los hechos para decidir sobre la aplicación al caso del art. 7.1, letra b RB I bis acudiendo a la interpretación realizada TJUE.

Palabras clave: competencia judicial internacional, competencia territorial, contrato de distribución, Reglamento Bruselas I bis.

Abstract: In a case concerning a distribution contract to be performed in Spain, the Court of Appeal of Asturias (Audiencia Provincial of Asturias) amends the decision of the first instance Court of Castropol to decline jurisdiction over a contractual claim on the ground that article 51 LEC should be applied as a consequence of Rome I Regulation. Dismissing this approach and considering that jurisdiction must be examined in the light of Brussels I Bis Regulation, the Court of Appeal carries out a twofold analysis: one focused on the scope of application of the BIR, and another on the applicability of the second paragraph of article 7(1)(b) BIR under the jurisprudence of the European Court of Justice.

Keywords: international jurisdiction, territorial jurisdiction, distribution contracts, Brussels I bis Regulation.
\end{abstract}

Sumario: I. Introducción. II. La identificación de la norma reguladora de la competencia judicial internacional de los tribunales españoles a través del ámbito de aplicación del Reglamento Bruselas I bis. III. Los conceptos autónomos del Reglamento en los litigios relativos a los contratos de distribución: la aplicación del art. 7.1, letra b, segundo guion al caso. 1. El concepto de contrato de prestación de servicios. 2. La determinación del lugar de prestación de los servicios. IV. Competencia judicial internacional y competencia territorial para los contratos de distribución. Conclusiones. 


\section{Introducción}

1. La determinación de la competencia judicial internacional a través de la aplicación del Reglamento Bruselas I bis todavía parece una tarea complicada para ciertos órganos judiciales españoles. Una muestra de la dificultad en la aplicación de esta norma del sistema europeo de Derecho Internacional Privado es la sentencia de la Audiencia Provincial de Oviedo de 25 de mayo de 2020 que debe estimar el recurso de apelación planteado contra la estimación de la declinatoria de competencia judicial internacional del Juzgado de Primera Instancia de Castropol -solicitada por el demandado en un caso de un contrato de distribución internacional-.

2. La Audiencia Provincial corrige los errores básicos de Derecho Internacional Privado en los que se incurre en primera instancia al determinar su competencia judicial internacional aplicando normas sobre la ley aplicable (Reglamento Roma I) y sobre la competencia territorial (art. 51 LEC) ${ }^{1}$. $\mathrm{Y}$ en este sentido aclara explícitamente que la cuestión jurídica planteada no es la de determinar la ley aplicable al contrato sino la competencia judicial internacional para conocer de la controversia. Por eso, la Audiencia Provincial rechaza la aplicación del Reglamento Roma I y la referencia de la juzgadora en primera instancia al lugar de cumplimiento de la prestación que sirve de base a la demanda para determinar la competencia judicial. Asimismo, la Audiencia corrige la confusión de los conceptos de competencia territorial y competencia judicial internacional clarificando que el artículo 51 LEC no determina la competencia judicial internacional de los tribunales españoles y no resulta aplicable al caso ${ }^{2}$.

3. La sentencia de 25 de mayo de 2020 se refiere al litigio planteado por una sociedad de nacionalidad española y con domicilio en Castropol (Asturias) contra una sociedad búlgara con domicilio en Sofía en relación a un contrato de distribución en España de mercancías fabricadas en Bulgaria. La demanda tiene por objeto, por un lado, la declaración de resolución unilateral e injustificada del contrato de distribución por parte de la empresa búlgara y, por otro lado, que se le condene al abono de una indemnización por clientela y por los daños y perjuicios por el lucro cesante causados por la falta de preaviso. Asimismo, se solicita la devolución de la cantidad satisfecha por la mercancía no entregada.

\section{La identificación de la norma reguladora de la competencia judicial internacional de los tribu- nales españoles a través del ámbito de aplicación del Reglamento Bruselas I bis.}

4. Acertadamente la Audiencia -una vez identificada correctamente que es la competencia judicial internacional, la cuestión de Derecho internacional privado que se plantea en el supuesto- parte del objeto del litigio para determinar la norma de nuestro ordenamiento jurídico donde debe encontrar la respuesta. En efecto, tal y como ha establecido el TJUE reiteradamente, para decidir si los foros del Reglamento Bruselas I bis rigen la competencia judicial internacional, el juzgador europeo debe cuestionarse si el objeto del litigio entra en su ámbito de aplicación material ${ }^{3}$. Y así hace la Audiencia Provincial que examina la pretensión de la demanda para entender que se refiere a una relación de naturaleza contractual y, por tanto, incluida en el ámbito de aplicación material del Reglamento Bruselas I bis.

\footnotetext{
${ }^{1}$ Esta norma incluida en el Capítulo II denominado "De las normas para determinar la competencia", en la Sección 2, titulada "de la competencia territorial", indica en su párrafo primero que Salvo que la Ley disponga otra cosa, las personas jurídicas serán demandadas en el lugar de su domicilio. También podrán ser demandadas en el lugar donde la situación o relación jurídica a que se refiera el litigio haya nacido o deba surtir efectos, siempre que en dicho lugar tengan establecimiento abierto al público o representante autorizado para actuar en nombre de la entidad.

${ }^{2}$ SAP Oviedo 25 mayo 2020, ECLI: ES:APO:2020:451A, FD $2^{\circ}$.

${ }^{3}$ STJUE 3 octubre 2012, Schneider, C-386/12, ECLI:EU:C:2013:633, Sobre el ámbito de aplicación material del Reglamento Bruselas I bis, véase P., RogERson, “Article 1", en U. MAGNUS/P. MANKOWSKI, Brussels I bis Regulation European Commentaries on Private International Law, Ottoschmidt, Sellier, 2016, pp. 53-86, y A.L. Calvo Caravaca /J. Carrascosa GonzÁlez, (DIR), Litigación internacional en la Unión Europea I, Competencia judicial y validez de resoluciones en materia civil y mercantil en la Unión Europea. Comentario al Reglamento Bruselas I bis, Thomson-Aranzadi, 2017, pp. 133 y ss.

${ }^{4}$ SAP Oviedo 25 mayo 2020, ECLI: ES:APO:2020:451A, FD $3^{\circ}$.
} 
5. En segundo lugar, y, siguiendo el razonamiento lógico que debe realizarse en un litigio con elemento extranjero, una vez aclarado el ámbito de aplicación material del Reglamento Bruselas I bis, la Audiencia examina el ámbito de aplicación personal del Reglamento analizando el domicilio del demandado y las consecuencias resultantes de la aplicación de artículos 4.1 y 5.1 RB I bis. De la aplicación de esas dos disposiciones se deducen los foros de competencia judicial internacional que pueden justificar la competencia del juzgado de instancia en el caso. Así, entiende, como no podía ser de otro modo, que al tener el demandado su domicilio en un Estado miembro (Bulgaria) no puede alegarse contra él las normas procesales internas españolas. Así que en el caso analizado pueden aplicarse únicamente los foros del Reglamento para demandar ante los tribunales españoles a la empresa domiciliada en Sofía 5 . Como el demandado está domiciliado en un Estado miembro (Bulgaria), la competencia del juzgado de Castropol sólo puede fundarse, a falta de acuerdo de jurisdicción, en el art. 7.1 RB I bis ${ }^{6}$.

\section{Los conceptos autónomos del Reglamento en los litigios relativos a los contratos de distribu- ción: la aplicación del art. 7.1, letra b segundo guion al caso.}

6. Una vez delimitada la cuestión de Derecho Internacional Privado y la norma aplicable para darle solución, debe tratarse la correcta aplicación de los foros del Reglamento Bruselas I bis en el caso que nos ocupa.

La aplicación del foro específico para los litigios en materia contractual del artículo 7 RB I bis al caso debe realizarse en dos fases. La primera consiste en calificar el tipo de contrato (1) y la segunda determinar el lugar de prestación de los servicios (2).

\section{El concepto de contrato de prestación de servicios}

7. Siendo evidente que la demanda sobre la que debe resolver la Audiencia Provincial tiene naturaleza contractual ya que la acción del demandante se refiere a una obligación jurídica libremente consentida por una persona respecto a otra ${ }^{7}$ la única opción que tenía el tribunal para decidir sobre la competencia judicial del Juzgado de Primera Instancia de Castropol, dado que no consta acuerdo de jurisdicción ni el litigio recae sobre materia relativa a las competencias exclusivas del art. 24 RBI bis, es aplicar el apartado primero del artículo 7 RB I bis.

8. Esta disposición tiene una estructura dividida en tres apartados, letras a, b y c, debiendo interpretarse, como bien hace la Audiencia, en primer lugar, si el supuesto puede incluirse en uno de los dos guiones de la letra $b$. Y ello es así porque el Tribunal de Justicia ya ha afirmado que el foro previsto en la letra a se aplica de manera únicamente alternativa y supletoria de los previstos en los dos guiones de la letra $b^{8}$.

De modo que sólo en caso de que no se pueda calificar el contrato como un contrato de compraventa o de prestación de servicios de la letra $\mathrm{b}$ debe el juzgador aplicar la letra a por remisión de la $\mathrm{c}$ del art. 7.1 RBI bis ${ }^{9}$. De manera que razona adecuadamente la Audiencia planteándose, en primer lugar, si

\footnotetext{
${ }^{5}$ SAP Oviedo 25 mayo 2020, ECLI: ES:APO:2020:451A, FD $3^{\circ}$.

${ }^{6}$ La materia objeto del litigio no es materia regulada por las competencias exclusivas del art. 24 RBI bis y no se deduce de los escritos intercambiados entre las partes que hayan acordado un tribunal para dirimir sus controversias.

${ }^{7}$ STJUE 14 marzo 2013, Česká spořitelna, C 419/11, EU:C:2013:165, apdos. 45 a 47, y de 28 enero 2015, Kolassa, C 375/13, EU:C:2015:37, apdos. 37 y 39).

${ }^{8}$ Citando únicamente las más recientes, véase STJUE 14 julio 2016, Granarolo, C 196/15, ECLI:EU:C:2016:559, apdos. 30 y 31 y STJUE 8 marzo 2018, Saey Home Garden NV-SA c. Máquinas e Accesórios Insustriáis S.A., C-64/17, ECLI:EU:C:2018:173, apdo. 24.

${ }^{9}$ La letra c indica que cuando la letra b) no sea aplicable, se aplicará la letra a) y esta última dice que una persona domiciliada en un Estado miembro puede ser demanda. en materia contractual, ante el órgano jurisdiccional del lugar en el que se haya cumplido o deba cumplirse la obligación que sirva de base a la demanda.
} 
el objeto del litigio puede incluirse en uno de los dos guiones del art. 7.1, letra b relativos a un contrato de compraventa y de prestación de servicios (FD $\left.4^{\circ}\right)$.

9. Este foro especial para los contratos no contiene una definición de contrato de compraventa ni de prestación de servicios, por lo que en el pasado surgieron dudas sobre su interpretación. La doctrina destacó la dificultad de determinar si debía aplicarse el primer o el segundo guion del art. 7.1, letra b RB I bis y realizó esfuerzos de identificación de criterios para la aplicación de uno u otro ${ }^{10}$.

10. A la luz de la jurisprudencia del Tribunal de Justicia, las disposiciones del Reglamento deben interpretarse de manera autónoma, refiriéndose al sistema y los objetivos de éste ${ }^{11}$. La doctrina ha afirmado también la necesidad de esa noción autónoma e independiente de los conceptos manejados en los ordenamientos jurídicos internos de los Estados miembros ${ }^{12}$.

De modo que para la calificación del contrato en el que se basa la demanda la Audiencia acude, oportunamente, a las numerosas sentencias en las que se realiza la interpretación de la norma por el Tribunal de Justicia.

11. El Tribunal de Justicia ya ha indicado que, dado que en el art. 7.1, letra b primer guion del RB I bis ni en sus precedentes se define el concepto de contrato de prestación de servicios, esta disposición debe interpretarse a la luz de la génesis, los objetivos y el sistema de dicho Reglamento ${ }^{13}$.

El Tribunal ha establecido que hay partir de la obligación característica del contrato ${ }^{14}$. Asimismo, ha dejado claro el tribunal que el concepto de servicios implica que la parte que los preste debe llevar a cabo una determinada actividad como contrapartida de una remuneración ${ }^{15}$.

La sentencia Falco Privatstiftung and Thomas Rabitsch vino a clarificar esta exigencia para la aplicación del segundo guion de la letra $b$ indicando que no es suficiente con la obligación de no hacer. Por tanto, para determinar la existencia de una actividad el Tribunal ha exigido la ejecución de actos positivos, excluyendo la simple abstención.

La Audiencia realiza en el fundamento jurídico cuarto el ejercicio de calificación del contrato sobre el que versa la demanda en atención a la jurisprudencia del TJUE. Aplicando la jurisprudencia Falco Privatstiftung and Thomas Rabitsch y la jurisprudencia posterior en la que se precisa que el concepto de «servicios», para los contratos de distribución comercial, implica, como mínimo, que la parte que los presta lleve a cabo una determinada actividad como contrapartida de una remuneración ${ }^{16}$.

\footnotetext{
${ }^{10}$ P. De Miguel Asensio, "El lugar de ejecución de los contratos de prestación de servicios como criterio atributivo de competencia”, en , J. FORNER I DELAYGUA/C. GONZÁLEZ BEILFUSS/R. VIÑAS FARRÉ (COORDS.), Liber amicorum Alegría Borrás, Marcial Pons, 2013, p. 291, 299; F. GARCimartín Alférez, Derecho Internacional Privado, Thomson Reuters, 2012, pp. 91 y ss.; M.A. CeBrián SALVAT, "Competencia judicial internacional en defecto de pacto en los contratos de distribución europeos: el contrato de distribución como contrato de prestación de servicios en el reglamento 44", CDT, 5,1, 2013, pp. 125-138; A.L. Calvo Caravaca/J. Carrascosa González (DiR), Litigación internacional en la Unión Europea I, Competencia judicial y validez de resoluciones en materia civil y mercantil en la Unión Europea. Comentario al Reglamento Bruselas I bis, Thomson-Aranzadi, 2017, pp. 313 y ss. y A.L. Calvo Caravaca/J. Carrascosa GonzÁlez (DiR), Derecho Internacional Privado, vol. II, Comares, 2017, p. 916.

${ }^{11}$ STJUE 16 enero 2014, Kainz, C45/13, ECLI:EU:C:2014:7, apdo. 19 y STJUE 10 septiembre 2015, Ferho, C-47/14, ECLI:EU:C:2015:574, apdos. 62-65, entre otras.

${ }^{12}$ P. Mankowski, “Article 7”, en U. MAGNUS/P. MANKOWSKI, Brussels I bis Regulation European Commentaries on Private International Law, Ottoschmidt, Sellier, 2016, pp. 121-369, esp. p. 198. A.L. CALvo Caravaca/J. Carrascosa GonzÁLEZ (DIR), Litigación internacional en la Unión Europea I, Competencia judicial y validez de resoluciones en materia civil y mercantil en la Unión Europea. Comentario al Reglamento Bruselas I bis, Thomson-Aranzadi, 2017, pp. 313-

${ }^{13}$ STJUE 13 julio 2006, Reisch Montage, C-103/05, ECLI:EU:C:2006:47, apdo. 29; STJUE 14 diciembre 2006, ASML, C-283/05, ECLI:EU:C:2006:787, apdos. 16 y 22, y STJUE 3 mayo 2007, Color Drack, C-386/05, ECLI:EU:C:2007:262, apdo. 18 y STJUE 23 abril 2009, Falco Privatstiftung, Thomas Rabitsch y Gisela Weller-Lindhorst, C-533/07 ECLI:EU:C:2009:257, apdo. 21.

${ }^{14}$ STJUE 25 febrero 2010, Car Trim, C-381/08, ECLI:EU:C:2010:90 y STJUE 15 junio de 20147, Kareda, C-249/16, ECLI:EU:C:2017:472.

${ }^{15}$ STJUE 15 junio 2017, Kareda, C-249/16, ECLI:EU:C:2017:472, apdo. 35.

${ }^{16}$ STJUE de 14 de julio de 2016, Granarolo, C 196/15, ECLI:EU:C:2016:559, apdo. 37 y jurisprudencia citada. Y STJUE de 15 de junio de 2017, Kareda, C-249/16, ECLI:EU:C:2017:472, apdo. 35. STJUE 19 diciembre 2013 Corman-Collins,
} 
12. El Tribunal ha precisado también que para los contratos de concesión la prestación característica es la ejecutada por el concesionario quien al llevar a cabo la distribución de los productos del concedente toma parte en el desarrollo de su difusión ${ }^{17}$.

13. En cuanto a la remuneración, cuestión en la que no entra la Audiencia, el Tribunal de Justicia ha realizado una interpretación laxa al entender que no es necesario que esta se haga mediante el pago de una cantidad dineraria sino que es suficiente que se conceda al distribuidor una ventaja competitiva al otorgársele, por ejemplo, la exclusividad o beneficiarse de la publicidad, de los conocimientos técnicos, de acciones de formación o de facilidades de pago ${ }^{18}$.

Por tanto, se puede afirmar hoy día que existe jurisprudencia consolidada del Tribunal de Justicia que considera los contratos de distribución exclusiva cubierto por el segundo guion de la letra $b$ del art. 7.1 RB I bis y que debe darse por superada la polémica doctrinal sobre la interpretación de la disposición ${ }^{19}$.

14. La Audiencia, después de hacer referencia a la jurisprudencia anterior que configura la interpretación del art. 7.1 RB I bis hace mención escasa a los hechos relativos a las obligaciones asumidas por las partes en el contrato y, en cambio, desarrolla la configuración del contrato de distribución en nuestro ordenamiento jurídico (FD $5^{\circ}$ ). Quizás la razón pueda encontrarse en la dificultad de averiguar las concretas prestaciones a realizar por el distribuidor en España al tratarse de un contrato verbal y disponer, tan solo, de ciertas comunicaciones intercambiadas por los contratantes que hacen referencia a la obligación del demandante de distribuir las bebidas adquiridas en España.

\section{La determinación del lugar de prestación de los servicios}

15. La interpretación del Tribunal de Justicia de la norma ha exigido como requisito para su aplicación que el lugar de la prestación de los servicios pueda determinarse, consagrando una interpretación autónoma del concepto de lugar de cumplimiento hasta tal punto que si en el caso no puede determinarse éste la disposición no debe aplicarse ${ }^{20}$.

16. El Tribunal de Justicia ha establecido que el lugar de prestación de los servicios puede determinarse con cierta flexibilidad y así, en un caso de indemnización por la resolución de un contrato de concesión mercantil, es el del Estado miembro en el que se encuentra el lugar de prestación principal de los servicios, según se desprenda de las cláusulas del contrato, o, a falta de tales cláusulas, el del cumplimiento efectivo del contrato o, cuando sea imposible determinarlo sobre tal base, el del domicilio del prestador ${ }^{21}$.

C-9/12, ECLI:EU:C:2013:860, apdo. 38 y STJUE de 8 marzo 2018, Saey Home \& Garden NV/SA c. Lusavouga-Máquinas e Acessórios Industriais SA, C-64/17, apdo. 39.

${ }^{17}$ STJUE 19 diciembre 2013 Corman-Collins, C-9/12, ECLI:EU:C:2013:860, apdo. 38 y STJUE de 8 marzo 2018, Saey Home \& Garden NV/SA c. Lusavouga-Máquinas e Acessórios Industriais SA, C-64/17, apdo. 39.

${ }^{18}$ STJUE 19 diciembre 2013 Corman-Collins, C-9/12, ECLI:EU:C:2013:860, apdo. 39 y 40 y STJUE de 8 marzo 2018, Saey Home \& Garden NV/SA c. Lusavouga-Máquinas e Acessórios Industriais SA, C-64/17, apdo. 40.

${ }^{19}$ LÓPEz RodríGuez, "El contrato de distribución exclusiva o concesión comercial como contrato de prestación de servicios a efectos de la aplicación del foro contractual del RB I", La Ley UE, no 13, 2014, p. 37; CeBrí́n SAlvat, M.A., "Competencia judicial internacional en defecto de pacto en los contratos de distribución europeos: el contrato de distribución como contrato de prestación de servicios en el reglamento 44", CDT, 5,1, 2013, p. 125.

${ }^{20}$ STJUE 19 febrero de 2002, Besix, C-256/00, ECLI:EU:C:2002:99, apdo. 36 y la jurisprudencia anterior citada. DE MIGUEL ASENSIO, P., "El lugar de ejecución de los contratos de prestación de servicios como criterio atributivo de competencia", en FORNER I DELAYGUA, J./GONZÁLEZ BEILFUSS, C./VIÑAS FARRÉ, R. (COORDS.), Liber amicorum Alegría Borrás, Marcial Pons, 2013, pp.302-304.

${ }^{21}$ Para el caso de que la comercialización de productos debiera producirse en el mercado nacional de un tercer Estado, STJUE de 11 marzo 2010, Wood Floor Solutions Andreas Domberger, C-19/09, ECLI:EU:C:2010:137, y STJUE 8 marzo 2018, Saey Home Garden NV-SA c. Máquinas e Accesórios Insustriáis S.A., C-64/17, ECLI:EU:C:2018:173, apdo. 45 y MoRENO SÁnCHEZ-MoraledA, A. "La determinación de la competencia judicial internacional en un contrato de prestación de servicios 
Como reflejo de esa flexibilidad, en las sentencias más recientes dictadas, el Tribunal, para garantizar la proximidad entre el litigio y el órgano jurisdiccional, averigua si el lugar de comercialización de los productos coincide o no con el del establecimiento de alguna de las partes en el contrato ${ }^{22}$.

Por tanto, no existiendo duda respecto del Estado miembro en el que debía lugar de prestación de los servicios en el caso resuelto por la Audiencia y siendo España también el lugar del domicilio del prestador, puede concluirse que este lugar garantiza el vínculo de conexión más estrecho entre el contrato y el órgano jurisdiccional competente. Por ello, la Audiencia atribuye competencia acertadamente al Juzgado de Instancia de Castropol.

17. Como resultado de la aplicación del segundo guion del art. 7.1, letra b RBI bis al caso se debe considerar competente al tribunal del lugar del Estado miembro en el que se deben prestar los servicios, resultando irrelevante el lugar donde debieran cumplirse las concretas obligaciones litigiosas. Por tanto, resultaría irrelevante el lugar del pago de la indemnización solicitada en la demanda.

18. La correcta aplicación de la norma requiere, asimismo, la consideración del lugar de prestación de los servicios como un dato fáctico, no una cuestión jurídica. En virtud de la literalidad de la norma, este lugar debe ser el pactado por las partes en el contrato y de los datos que constan en el auto comentado relativos a las comunicaciones entre las partes se deduce que la demandante distribuía las mercancías en España, por lo que resulta evidente la atribución de competencia a los tribunales españoles. Sin embargo, la Audiencia se hace eco de la doctrina sentada por el TJUE conforme a la cual, si las partes no han pactado el lugar de prestación de los servicios, debe tenerse en cuenta el lugar donde se han prestado efectivamente, o de facto, los servicios. Esta doctrina ya establecida por el Tribunal de Justicia en el caso Ferho ${ }^{23}$, ha sido confirmada por la sentencia Saey Home Garden NV. La aplicación por la Audiencia del lugar del cumplimiento efectivo del contrato o, cuando sea imposible determinarlo sobre tal base, el del domicilio del prestador ${ }^{24}$ le permite atribuir competencia al Juzgado de Primera Instancia de Castropol, lugar del domicilio del distribuidor de las mercancías y demandante en el litigio.

\section{Competencia judicial internacional y competencia territorial para los contratos de distribu- ción}

19. El auto analizado pone de manifiesto también la necesidad de diferenciar dos conceptos básicos en Derecho Internacional Privado que han sido confundidos en instancia; la noción de competencia judicial internacional no debe equivocarse con la de competencia territorial. Esa diferencia ha sido señalada desde tiempo inmemorial por la doctrina de Derecho Internacional Privado ${ }^{25}$.

20. Esta diferenciación debe realizarse también en los litigios relativos a los contratos internacionales de distribución en los que debe determinarse tanto la competencia judicial internacional y la territorial.

entre Estados miembros. Comentario a la Sentencia del Tribunal de Justicia de la Unión Europea, Sala Séptima, de 8 de marzo de 2018. Caso Saey Home \& Garden NV/SA contra Máquinas e Acessórios Industriais, S.A.”, Revista de Derecho Patrimonial num.46/2018, ECLI:EU:C:2018:173

${ }^{22}$ STJUE de 8 marzo 2018, Saey Home \& Garden NV/SA c. Lusavouga-Máquinas e Acessórios Industriais SA, C-64/17, ECLI:EU:C:2018:173.

${ }^{23}$ STJUE de 10 septiembre 2015, Ferho, C-47/14, ECLI:EU:C:2015:574, apdos. 62-65.

${ }^{24}$ STJUE de 8 marzo 2018, Saey Home \& Garden NV/SA c. Lusavouga-Máquinas e Acessórios Industriais SA, C-64/17, ECLI:EU:C:2018:173, apdo. 47.

${ }^{25}$ Calvo Caravaca, A.L./Carrascosa González, J., Derecho Internacional Privado, vol. I, Comares, 2018, pp. 143-146 y Compendio de Derecho Internacional Privado, 2020, p. 42. 
La Audiencia repasa la numerosa jurisprudencia del Tribunal de Justicia que, interpretando el artículo 7.1, letra b indica que es un foro de competencia tanto internacional como territorial ${ }^{26}$. En el caso Besix se establece que la aplicación de esta disposición debe designar al juez territorialmente más idóneo para conocer de un concreto litigio ya que de lo contrario se estaría vulnerando el principio de seguridad jurídica ${ }^{27}$.

En la misma línea, en el caso, Kareda, el Tribunal afirma que la norma debe interpretarse en el sentido de que, cuando una entidad de crédito ha concedido un préstamo a dos codeudores solidarios, el «lugar del Estado miembro en el que, según el contrato, hayan sido o deban ser prestados los servicios», a efectos de este precepto, es, salvo pacto en contrario, el del domicilio de la entidad, y asimismo lo es a efectos de determinar la competencia territorial del órgano jurisdiccional que haya de conocer de la acción de repetición entre dichos codeudores ${ }^{28}$. El Tribunal afirma, asimismo, que de los objetivos de previsibilidad, unificación y recta administración de justicia se deriva que la interpretación del lugar de prestación de los servicios lo es también a la hora de determinar la competencia territorial ${ }^{29}$.

21. La Audiencia acude muy correctamente a la jurisprudencia del Tribunal de Justicia en el caso Color Drack en un caso de compraventa de mercancías a entregar en diversos lugares dentro de un mismo Estado miembro. Al aplicar el primer guion del art. 5.1 de la letra b, el Tribunal de Justicia afirma que dicha disposición determina tanto la competencia internacional como la territorial, esta disposición tiene por objeto unificar las reglas de conflicto de jurisdicción y, por consiguiente, determinar directamente el foro competente sin realizar una remisión a las reglas internas de los Estados miembros ${ }^{30}$. En el caso Color Drack y el resuelto por la Audiencia Provincial de Oviedo coincidía que el domicilio social de una de las partes -demandado en el primero y demandante en el segundo- se encontraba en la demarcación del tribunal ante el que se había planteado la demanda ${ }^{31}$.

La Audiencia concluye, como no podía ser de otro modo, que el segundo guion es un foro de competencia tanto internacional como territorial, de modo que no es necesario acudir a las normas internas de los Estados miembros (en nuestro caso el art. 51 LEC) para determinar el tribunal competente.

22. En el caso que nos ocupa, la solución aplicada por la Audiencia responde a un objetivo de proximidad, motivada por la existencia de un estrecho vínculo de conexión entre el contrato y el tribunal que debe conocer del mismo tal y como exige el Tribunal de Justicia en el caso Color Drack ${ }^{32}$.

\section{Conclusiones}

Primera: Los errores cometidos por el Juzgado de Primera Instancia de Castropol afectan al derecho fundamental de la tutela judicial efectiva ya que ocasionan que se declare incompetente. De no haberse corregido la errónea aplicación de los conceptos y de las normas europeas de Derecho Internacional Privado por la Audiencia se hubiera obligado al demandante domiciliado en España, en un litigio relativo a un contrato que se ejecutó en España a acudir a los tribunales búlgaros a reclamar sus derechos. Este caso refleja que, con el fin de no incurrir en denegación de justicia, continúa siendo necesario incidir en el aprendizaje de los conceptos básicos de Derecho Internacional Privado y en la adecuada aplicación de sus normas de origen internacional.

\footnotetext{
${ }^{26}$ Por tanto, en España no debe aplicarse el art. 51 LEC, Calvo Caravaca, A.L./CArrascosa GonzÁlez, J. (Dir), Litigación internacional en la Unión Europea I, Competencia judicial y validez de resoluciones en materia civil y mercantil en la Unión Europea. Comentario al Reglamento Bruselas I bis, Thomson-Aranzadi, 2017, p. 300.

${ }^{27}$ STJUE 19 febrero de 2002, Besix, C-256/00, ECLI:EU:C:2002:99, apdo. 35.

${ }^{28}$ STJUE de 15 junio de 2017, Kareda c. Benkö, C-249/16, ECLI:EU:C:2017:472, apdos. 44 y 46.

29 Ibidem, apdo. 46.

${ }^{30}$ STJUE de 3 de mayo de 2007, Color Drack, C-386/05, ECLI:EU:C:2007:262, apdo. 30. En este caso se trataba de un litigio relativo a un contrato de compraventa y se analizó, por tanto, la aplicación del primer guion del art. 7.1. letra b del Reglamento 44/2001 pero sus afirmaciones son trasladables al contrato de prestación de servicios en el Reglamento vigente.

${ }^{31}$ STJUE de 3 de mayo de 2007, Color Drack, C 386/05, ECLI:EU:C:2007:262, apdo. 10.

${ }^{32}$ Ibidem, apdo. 22.
} 
Segunda: La interpretación realizada por la Audiencia del artículo 7.1, letra b RB I bis y su aplicación al caso respeta los principios de interpretación restrictiva y la garantía de la previsibilidad y protección del demandado como exige el TJUE.

Tercera: La sociedad demandada con domicilio en Bulgaria debe asumir el riesgo de la internacionalidad y defenderse ante los tribunales españoles, tribunales del lugar de distribución de las mercancías objeto del contrato, que debe ser considerado un foro previsible. La aplicación del art. 7, apartado primero RB I bis realizada por la Audiencia Provincial al caso cumple, por tanto, con los objetivos del Reglamento de previsibilidad y proximidad del tribunal con el litigio. 